\title{
COVERAGE OPTIMIZED AND TIME EFFICIENT LOCAL SEARCH BETWEENNESS ROUTING FOR HEALTH MONITORING IN WSN
}

\author{
M. Parthasarathi ${ }^{1}$ and Karthikeyani Vajravel ${ }^{2}$ \\ ${ }^{1}$ Department of MCA, KSR College of Arts and Science, \\ Tiruchengode, Namakkal - Dt \\ and \\ ${ }^{2}$ Department of Computer science, \\ Thiruvalluvar Govt Arts college, Rasipuram, Namakkal Dt.
}

\begin{abstract}
Wireless Sensor Networks has been widely used for monitoring and control applications in our daily life due to its appealing characteristic like low cost, power efficient, easy to implement in many areas such as war zone, medical monitoring optimization and so on. However, Wireless Sensor Networks (WSN) requires a new routing algorithm for health monitoring. In this paper, an efficient routing model called Local Search and Enhanced Betweenness Routing (LS-EBR) in WSN to improve routing efficiency by increasing sensor nodes coverage and minimizing the time for routing is presented. The LS-EBR model in WSN for health monitoring uses enhanced betweenness routing that measures energy consumption of the neighboring nodes in a local search manner. First, a novel Local Search model that does not require global information about the entire network and in turn divert routing from sensor nodes that are more frequently used is used. The aim is to reduce the time for routing. Second, an Enhanced Betweenness Routing model that not only considers routing overhead but also considers the remaining energy of sensor node into account to ensure higher number of sensor nodes to be monitored, thus achieving much higher coverage is designed. Finally, working together with the above Local Search and Enhanced Betweenness Routing by applying Enhanced Betweenness Routing algorithm, reduces the average energy consumed. Simulation results showed that the proposed routing model has advantages over opportunistic routing with respect to coverage and improving routing efficiency by reducing time for routing in wireless sensor networks.
\end{abstract}

\section{KEYWORDS}

Wireless Sensor Network, Local Search, Betweenness Routing, Health Monitoring

\section{INTRODUCTION}

In wireless sensor networks, due to the limited non-rechargeable battery power, routing protocols attempt to find effective routing mechanism to be followed in order to reach the sink node. Many research works have been conducted in this area.

Opportunistic Routing for Relay Node Selection (OR-RNS) [1] in WSN provided an insight into energy optimization during routing by applying energy equivalent node. A distributed Adaptive Opportunistic Routing (AOR) [2] scheme was applied with the objective of improving the DOI:10.5121/ijcsa.2016.6104 
alternative routes in the network. Another energy optimized model with efficient routing mechanism called, Least Cost Any Path (LCAP) [3] routing was applied with the objective of improving the robustness during routing. On the other hand, with the objective of maximizing the network throughput, in [4], routing and code division channelization was applied to reduce the delay performance during routing.

Routing in addition to privacy is receiving maximum attention due to the open nature and mobility of wireless media. In [5], unobservable secure routing scheme was designed using group signature and ID-based encryption with the objective of providing a strong privacy mechanism for the packets being transmitted in the network.

Several studies have shown that packet receptions on wireless links are correlated. Link aware correlation opportunistic routing algorithm was studied in [6] to improve the number of transmission rates. In [7], optimal routing metric was designed for cognitive radio ad hoc networks. In [8], optimization mechanism was designed to provide an opportunistic routes and rate limits by applying broadcast loss model. Geographic routing is attractive due to the fact that the routing state needed for packet forwarding is independent of network size. Multihop Delaunay Triangulation [9] was applied with the objective of improving the routing success rate for 3D and 4D views. Correlation aware QoS routing algorithm [10] was designed to efficiently deliver visual information for wireless video sensor network with the aid of inter-node coding differential scheme.

In this paper, we investigate the problem of coverage optimized and time efficient routing model in wireless sensor networks in a local search manner. We propose a Local Search and Enhanced Betweenness Routing (LS-EBR) in WSN which minimizes the time for routing a packet from a source node to the sink node. Specifically, an Enhanced Betweenness Routing algorithm for an increased coverage and minimum energy consumption is developed. The performance characteristics are demonstrated by simulation studies.

The remainder of the paper is organized as follows. Section 2 presents related work. Section 3 explains the Local Search and Enhanced Betweenness Routing model for health monitoring on wireless sensor networks. Section 4 evaluates the performance of LS-EBR model by simulation. Section 6 concludes the paper.

\section{RELATED WORKS}

There were several prior proposals to apply time efficient and energy efficient routing algorithm. Orthogonal Variable Spreading Factor (OVSF) [11] based routing protocol was applied to improve network lifetime and reduce the end to end delay. An energy optimized routing based on fuzzy logic for wireless sensor network using multi hop forwarding scheme was presented in [12]. In order to improve routing in wireless sensor network, another method based on Bayes node energy polynomial distribution was presented in [13].

With the increase in growth in healthcare industry and rise in its costs, considerable attention has been given to the structural health monitoring. Minimum hop count and link cost function [14] were applied to improve the packet delivery ratio and reduce the end to end delay by selecting the next hop node. Monitoring global invariants [15] in wireless sensor network was addressed for distributed monitoring. To improve the network lifetime, a routing protocol for many-to-many 
communication was presented in [16]. Another routing protocol to improve the network lifetime was presented in [17] by selecting vice cluster head using LEACH protocol.

Due to the ubiquitous nature of the network, wireless communication can be deployed with large populations. In [18], dynamic energy based relaying was applied in both one-dimensional and two-dimensional networks based on linear programming to achieve improved network lifetime. Issues in healthcare perspective were analyzed in [19]. On the basis of health care perspective, data aggregation scheme for large scale wireless network was presented in [20].

If we only consider the energy efficiency routing and ignore other aspects like time taken to identify optimized routing, it is not efficient on the performance improvement of WSNs. Thus we propose a new Local Search and Enhanced Betweenness Routing (LS-EBR) model in WSN to not only reduce the time for routing but also to improve the coverage with minimum energy consumptions.

\section{PROPOSED METHOD}

The network model, initialization of sensor node, Local Search based on Greedy Forwarding, and the Enhanced Betweenness Routing model for Wireless Sensor Networks are discussed in the following subsections.

\subsection{Network Model}

Let us consider a wireless sensor network that sense the patient with the aid of connectivity graph denoted as ' $G=(V, E)$ '. Here ' $V \in S$ ' and ' $S$ ' represents the set of all sensor deployed that track patient located at random position given as ' $S=S_{1}, S_{2}, \ldots, S_{n}$ ' at time ' $T$ '. On the other hand, ' $E$ ' represents the wireless link between any two sensor nodes denoted as ' $E=e_{1}, e_{2}, \ldots, e_{3}$ ', respectively. The sensor nodes not only perform the task of sensing but also act as broadcasting nodes for forwarding each other's data within a transmission range ' $R$ ', following a Poisson model with number of sensor nodes in WSN with ' $N(R)$ ' as given below.

$$
\text { Poisson model (Transmission Range }(r))=\exp ^{-\lambda N R} \frac{\lambda \mid N R * L}{L !}
$$

From (1), ' $\lambda$ ' represents the probability that a single patient is present in a monitoring region whereas ' $N(R)$ ' represents the transmission range to be monitored. It is the goal of this paper to design an efficient routing mechanism in such a way to reduce the average time for routing and improve the coverage or maximum number of sensor nodes being monitored using Local Search and Enhanced Betweenness Routing in WSN.

\subsection{Sensor Node Initialization Phase}

In this Sensor Node Initialization phase, each sensor node in WSN is informed with its neighbor nodes, sink node positioning and the possible routes to sink node. Figure 1 shows a structure of wireless sensor network. 


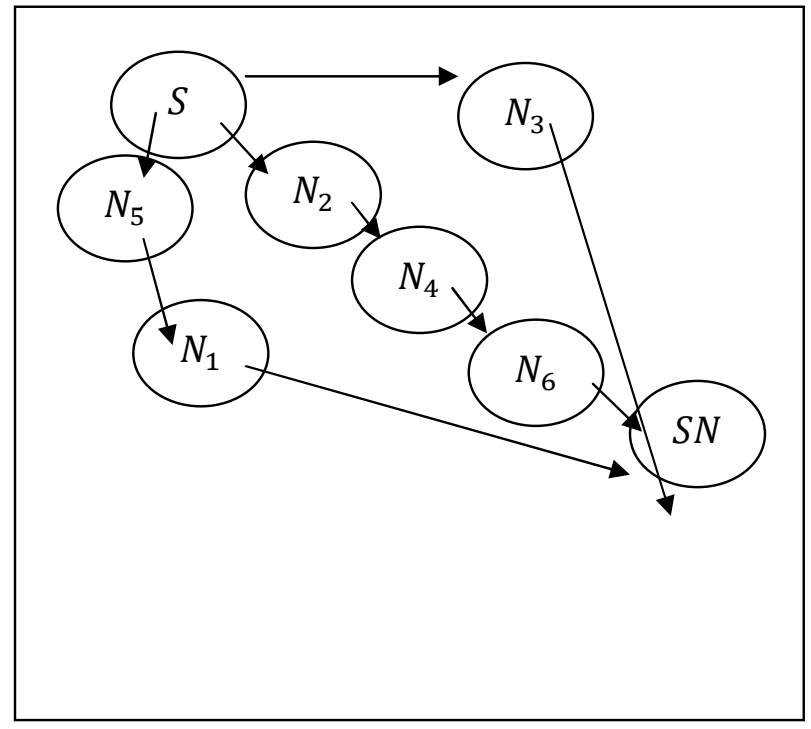

As shown in the figure, each sensor node can sense the object within a fixed transmission range ' $R$ ' and also forwards packets. Upon reception of a HELLO message, the sensor nodes in the network update their routing table. The figure shows a sensor node ' $S$ ' with six neighboring nodes ' $N_{1}, N_{2}, N_{3}, N_{4}, N_{5}, N_{6}$ ' Figure 2 shows the structure of the HELLO message format.

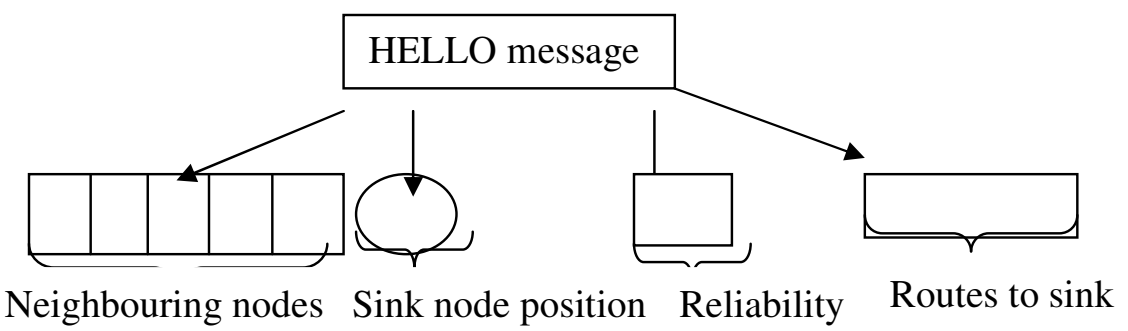

Figure 2 structure of HELLO message

As shown in the figure as soon as a HELLO message is received in the network, the neighboring nodes of the node that sent the message, the positioning of the sink nodes, the reliability of the neighboring nodes and the possible routes from the source sensor node to the sink node is obtained. This information is stored in the routing table (figure 3).

\begin{tabular}{|c|l|l|l|}
\hline Sensor node & Neighboring nodes & Sink node position & Routes to sink node \\
\hline \multicolumn{1}{|c|}{$S$} & ' $N_{1}, N_{2}, N_{3}, N_{4}, N_{5}, N_{6}$ ' & \multicolumn{2}{c|}{$N_{2}, N_{4}, N_{6}}$, \\
& & & ' $N_{5}, N_{1},{ }^{\prime} N_{3}$, \\
\hline---- & ---- & ---- & ---- \\
\hline---- & ---- & ---- & ---- \\
\hline
\end{tabular}

Figure 3 Routing table 
International Journal on Computational Science \& Applications (IJCSA) Vol.6,No.1,February 2016

The sensor nodes as shown in figure 1 update the routing table whenever a sensor node sends HELLO message. Once the sensor node initialization is accomplished, local search is performed which is explained in the next section.

\subsection{Greedy Forwarding-based Local Search}

Upon construction of a routing table, the next step towards the design of Local Search and Enhanced Betweenness Routing (LS-EBR) model in WSN is to construct a local search algorithm based on Greedy Forwarding. Given a wireless sensor network that comprises of ' $n$ ' sensor nodes randomly distributed over an area of ' $N * N$ meters', the sensor nodes lying within a transmission range ' $R$ ' send a short message to the sink node ' $S N$ ' containing information of their respective geographical locations.

Therefore, whenever a patient object enters into the network, the sensor nodes in the network senses the objects and sends the detected object to the sink node ' $S N$ '. On the other hand, the sink node aggregates the objects event according to the event detected. The detected event may be glucose level monitoring, heart rate monitoring, home monitoring and so on. These events have to be routed to the sink node. Several methods exist in performing efficient routing.

In this work, Local Search is applied which is based on greedy forwarding. The principle behind Greedy Forwarding is to perform a local search by forwarding the packets to its neighbor node that is closest to the destination node. Therefore, each sensor node (i.e. patient object) forwards the message to the neighbor node considering a local view, which can be the one that reduces the routing time to reach the destination in each step.

As the LS-EBR model in WSN applies greedy forwarding to find a route from the source sensor to the sink node, the optimum next sensor node forwards the data packet to a neighbouring node that should not only be closest to the destination node and has sufficient end-to-end path reliability to forward the data packet.

In order to construct a closest function to determine the node closest to the destination node, let us consider that a sensor node ' $S N_{j}$ ' has neighbour nodes ' $N_{1}, N_{2}, \ldots, N_{n}$ ' and destination node ' $D$ ' respectively that transmits packet ' $P_{i}$ '. Let us consider that the information in the packet include blood pressure of a patient. Then, the closest function used to determine the node closeness to the destination node is given as below.

$$
C F=M I N\left(d i s_{1}, d i s_{2}, \ldots, d i s_{n}\right)
$$

From (2) ' $d i s_{i}$ ', symbolizes the distance between the two neighbouring nodes (i.e. the new object being detected and their neighbouring node respectively). The distance ' $d i s_{i, j}$ ' is evaluated as given below.

$$
d i s_{i, j} \rightarrow \sqrt{\left(i_{1}-j_{1}\right)^{2}+\left(i_{2}-j_{2}\right)^{2}}
$$

Followed by the closest function obtained, the value of reliability is measured on the basis of the ratio of number of successful transmission made by the sensor node ' $T_{\text {succ }}\left(S N_{i}\right)$ ' to the total 
number of transmission made by the corresponding sensor node ' $T_{\text {total }}\left(S N_{i}\right)$ ' and is as given below.

$$
R\left(S N_{i}\right)=\frac{T_{\text {succ }}\left(S N_{i}\right)}{T_{\text {total }}\left(S N_{i}\right)}, \text { where } i=1,2, \ldots, n
$$

From (4), higher the value obtained, more efficient is the reliability of the sensor node is said to be. Initially, the reliability of sensor node is set to be zero in the routing table. Only after transmission, the value of reliability in the routing table gets changed. Figure 4 shows the Local Search algorithm.

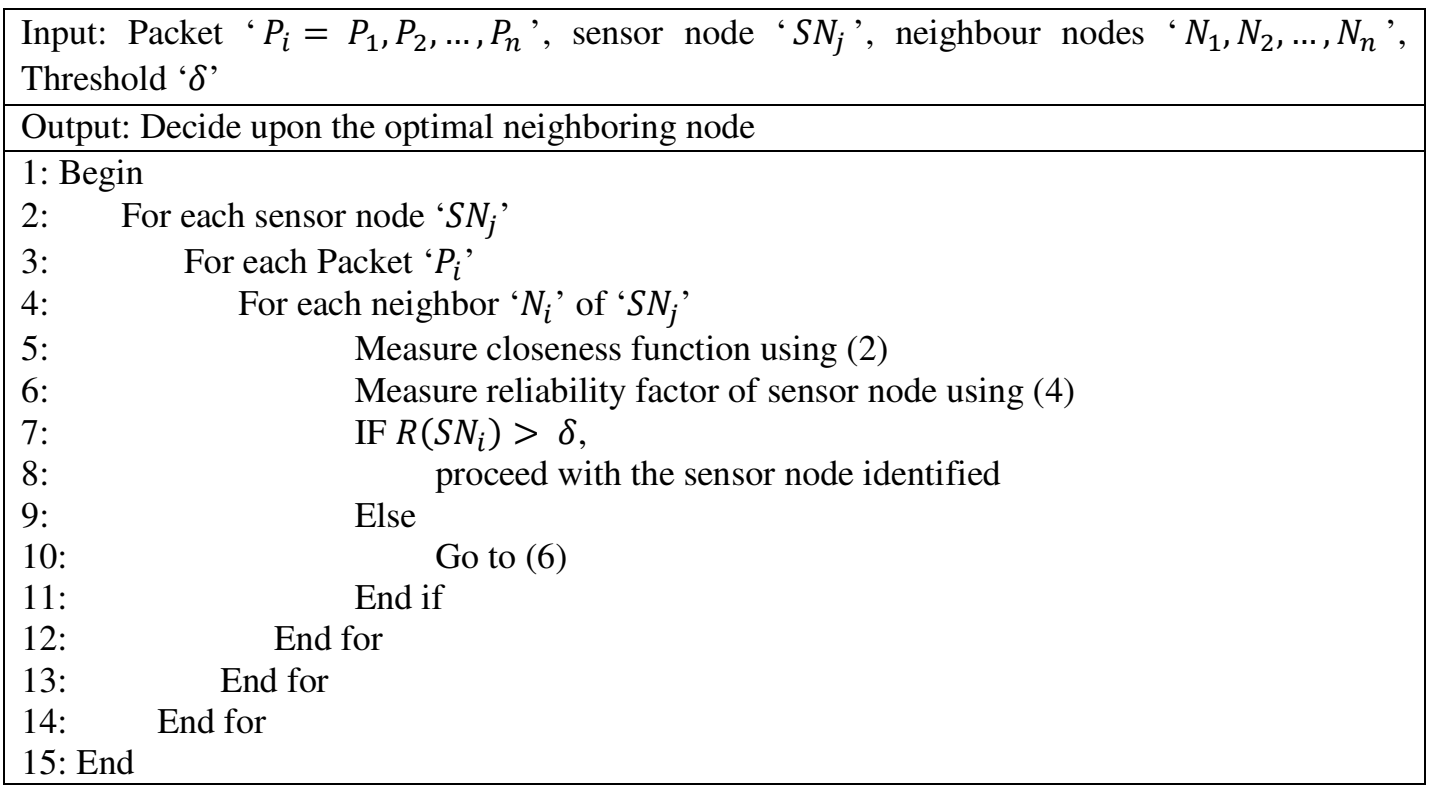

Figure 4 Local Search Routing

As shown in the figure 4, the local search algorithm based on greedy forwarding not only identifies the neighboring node closest to the destination but also measures the reliability of the sensor node. When a sensor node sends a packet (that contains the blood pressure information of a patient), the objective of local search algorithm is to identify the closest node to the destination node using only local information.

Once the closeness factor is measured, the next step is to determine the reliability of the sensor node. The reliability obtained is compared with the threshold value. If the resultant value of reliability is greater than the value of the threshold, the sensor node is considered as the closest node and reliable node. Otherwise, the sensor node is not considered to be a reliable node and the process proceeds until identification of the reliable node is made. In this way the local search algorithm with the local information minimizes the time for routing.

\subsection{Enhanced Betweenness Routing Model}

With the objective of detecting routing model for dense wireless sensor networks, a Betweenness Routing model that considers routing overhead and remaining energy of sensor node is taken into 
account. The routing overhead considers the average number of routing packets transmitted to deliver a packet, where each hop transmission is counted as one routing packet.

$$
A R O=\frac{H O P_{j p}}{\text { Total packets }}
$$

From (5), ' $j$ (packets)' symbolizes the packets represented as ' $P_{1}, P_{2}, \ldots, P_{n}$ ' whereas ' $p$ ' symbolizes the path represented by ' path $_{1}$, path $_{2}, \ldots$, path $_{n}$ ' respectively. Assuming that the sensor node obtaining a route for forwarding a data packet consumes energy ' $E_{R}$ ' is obtained by combining (5) and (3)

$$
E_{R}=\sqrt{\left(i_{1}-j_{1}\right)^{2}+\left(i_{2}-j_{2}\right)^{2}} * \frac{H P_{j p}}{\text { Total packets }}
$$

From (6), the routing overhead and remaining energy of sensor node is obtained and the route with minimum overhead and minimum energy consumed by a sensor node is used as a route path. The Enhanced Betweenness Routing algorithm is shown in figure 5.

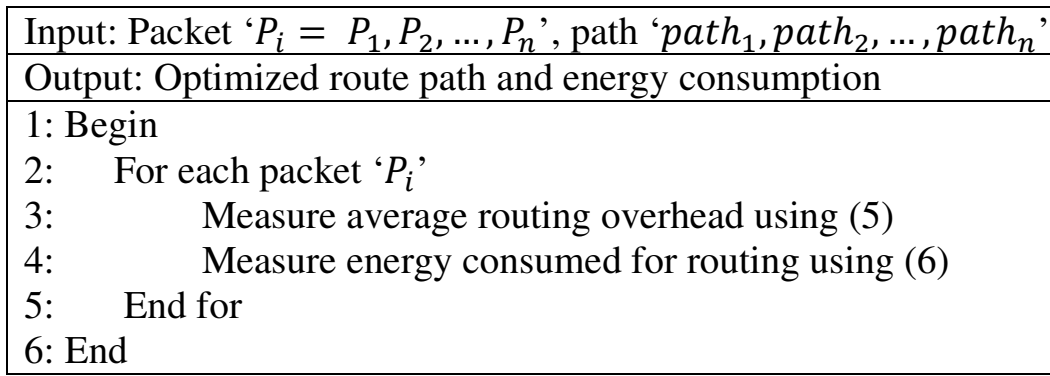

Figure 5 Enhanced Betweenness Routing algorithm

As shown in the figure 5, the Enhanced Betweenness Routing, measures the average routing overhead between all pairs of vertices by restricting the length of routing overhead. In the enhanced betweenness routing algorithm, instead of considering the shortest path, we measure the average routing overhead and the remaining energy consumed by the sensor node in the network. In this way, by applying Enhanced Betweenness Routing model higher coverage is addressed.

\section{SIMULATION SETUP AND PERFORMANCE METRICS}

In this section, we present our simulation setup for Local Search and Enhanced Betweenness Routing (LS-EBR) in WSN, followed by the performance metrics, comparisons and discussions with state-of-the-art works.

\subsection{Simulation Setup}

We have conducted an extensive simulation study using NS2 to evaluate the performance of our routing model for various performance metrics. We compared our proposed LS-EBR model with the Opportunistic Routing algorithm for Relay Node Selection (OR-RNS) [1] which also 
considers energy saving and shows the performance of LS-EBR to be comparatively better. Also we compare our proposed routing model with an Adaptive Opportunistic Routing scheme (AOR) [2] and measure the efficiency our LS-EBR.

A fair comparison is said to be achieved only with careful selection of simulation parameters. The performance evaluation of our routing model is done by considering a network where 70 to 240 nodes are randomly scattered in a field of $1200 \mathrm{~m} \times 1200 \mathrm{~m}$ area. We further assume that all the sensor nodes in the network are static after deployment with transmission range of $60 \mathrm{~m}$.

The simulation parameters used are as follows. Simulation time is set to $500 \mathrm{sec}$ and the size of a data packet is 128 bytes with a fixed generation rate of 1 packet/sec. Source nodes are picked in a random manner whereas the sink positioning is fixed towards the top left side of the simulation area. The results of simulation are obtained from several configurations and multiple runs, and the results shown are averaged over 7 simulation runs.

\subsection{Performance metrics}

Routing time measures the time taken to obtain efficient routing. Lower the routing time, more efficient the method is said to be and is formulated as given below. Time taken for routing is the difference between the end time and start time for routing by the sensor node in WSN. It is measured in terms of milliseconds and is formulated as given below.

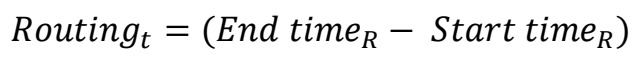

From (7), the time for routing is measured using 'Routing ${ }_{t}$ ', where 'End time ${ }_{R}$ ' represents the end time for routing by the sensor node and 'Start time ${ }_{R}$ ' represents the start time for routing by the sensor node in WSN.

Coverage: Sensing coverage is one of the important issues in wireless sensor networks. It also refers to the number of sensor nodes monitored at a time interval ' $t$ '.'

$$
C=\sum_{i=1}^{n} \frac{\left(S N_{i}-S N_{n m}\right)}{10} * 100
$$

From (8), ' $C$ ' symbolizes the coverage, ' $S N_{i}$ ' the number of sensor nodes in the network with ' $S N_{n m}$ ' representing the sensor nodes not monitored at a time interval ' $t$ '.

Average energy consumption: The average energy consumption per transmission in a way serves as a measure for network lifetime; less energy consumption per transmission indicates more network lifetime and vice versa. The average energy consumption measured is as given below.

$$
A E C=\frac{\text { Total energy consumption }}{\text { Number of packets received }}
$$

\section{DISCUSSION}

In this section the result analysis of LS-EBR model is made and compared with two existing methods, Opportunistic Routing algorithm for Relay Node Selection (OR-RNS) [1] and an 
Adaptive Opportunistic Routing scheme (AOR) [2] in WSN. The nodes in LS-EBR model are positioned in uniform topology. To evaluate the efficiency of LS-EBR model, the following metrics like routing time, coverage and average energy consumption for routing in Wireless Sensor Network is measured.

We begin by examining the effects of routing time of the proposed LS-EBR model using the same values as in table 1 and the number of sensor nodes is set to 70 . To highlight the ability of LS-EBR model to distinguish services in terms of routing time, figure compares the probability of achieved routing time with respect to different node density.

Table 1 Routing time comparisons

\begin{tabular}{|c|c|c|c|}
\hline \multirow{2}{*}{$\begin{array}{c}\text { Sensor node } \\
\text { density }\end{array}$} & \multicolumn{3}{|c|}{ Routing time (ms) } \\
\cline { 2 - 4 } & LS-EBR & OR-RNS & AOR \\
\hline 10 & 128 & 152 & 175 \\
\hline 20 & 185 & 205 & 224 \\
\hline 30 & 249 & 274 & 293 \\
\hline 40 & 295 & 320 & 339 \\
\hline 50 & 348 & 373 & 392 \\
\hline 60 & 421 & 446 & 465 \\
\hline 70 & 496 & 520 & 539 \\
\hline
\end{tabular}

The values obtained through () is tabulated for different sensor node density using the proposed LS-EBR model and compared elaborately with the existing two works OR-RNS [1] and AOR [2] respectively.

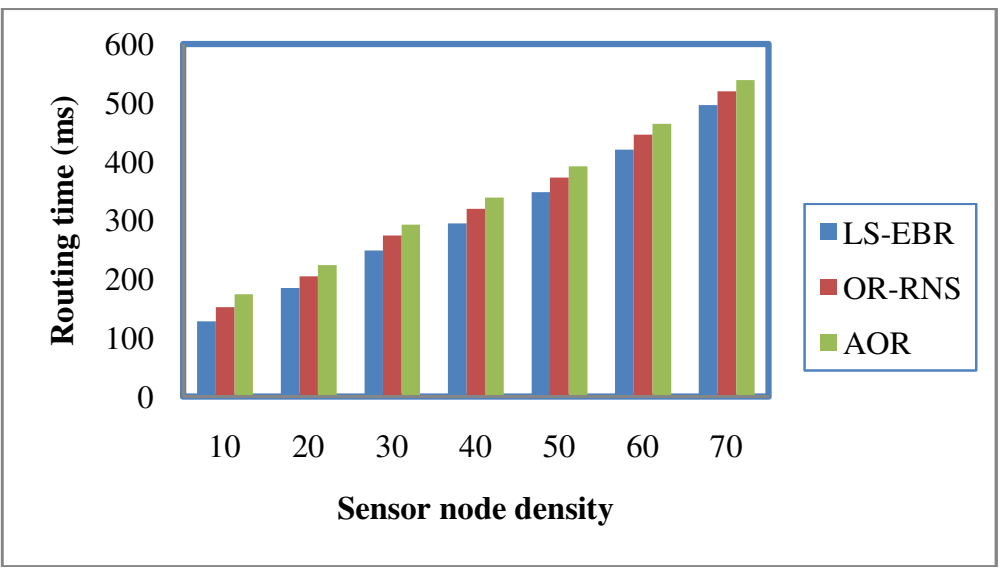

Figure 6 Routing time with different node density 
International Journal on Computational Science \& Applications (IJCSA) Vol.6,No.1,February 2016

Figure 6 illustrates the routing time required for various numbers of nodes that considered the end time and starting time for routing in LS-EBR model and compared with OR-RNS [1] and AOR [2] respectively. In LS-EBR model, the routing time is outperformed compared to the two other existing methods. The results reported above confirm that with the increase in the number of sensor nodes in WSN, various routes are available to reach the sink node.

Therefore, optimized route is selected in LS-EBR model by applying Enhanced Betweenness Routing model. So, the time for routing obtained is said to be comparatively lesser than $9 \%$ using LS-EBR when compared to OR-RNS. In addition, the Enhanced Betweenness Routing model in LS-EBR not only considers the average routing overhead but also the remaining energy in sensor network while routing. This makes the LS-EBR model to reduce the routing time by $17 \%$ compared to AOR.

The coverage for different node density with a range of 10 to 70 nodes are considered and the results of seven simulation runs conducted to measure the coverage rate are listed in table 2 . As listed in table 2, the LS-EBR model measures the coverage in terms of percentage (\%). The coverage obtained using our model LS-EBR offer comparable values than the state-of-the-art methods.

Table 2 Coverage comparisons

\begin{tabular}{|c|c|c|c|}
\hline \multirow{2}{*}{ Sensor node density } & \multicolumn{3}{|c|}{ Coverage (\%) } \\
\cline { 2 - 4 } & LS-EBR & OR-RNS & AOR \\
\hline 10 & 93.15 & 85.23 & 74.24 \\
\hline 20 & 85.14 & 78.22 & 67.17 \\
\hline 30 & 82.13 & 74.21 & 63.16 \\
\hline 40 & 84.29 & 76.37 & 65.32 \\
\hline 50 & 86.17 & 78.25 & 67.20 \\
\hline 60 & & & 69.26 \\
\hline 70 & 98.23 & 80.31 & 76.89 \\
\hline
\end{tabular}




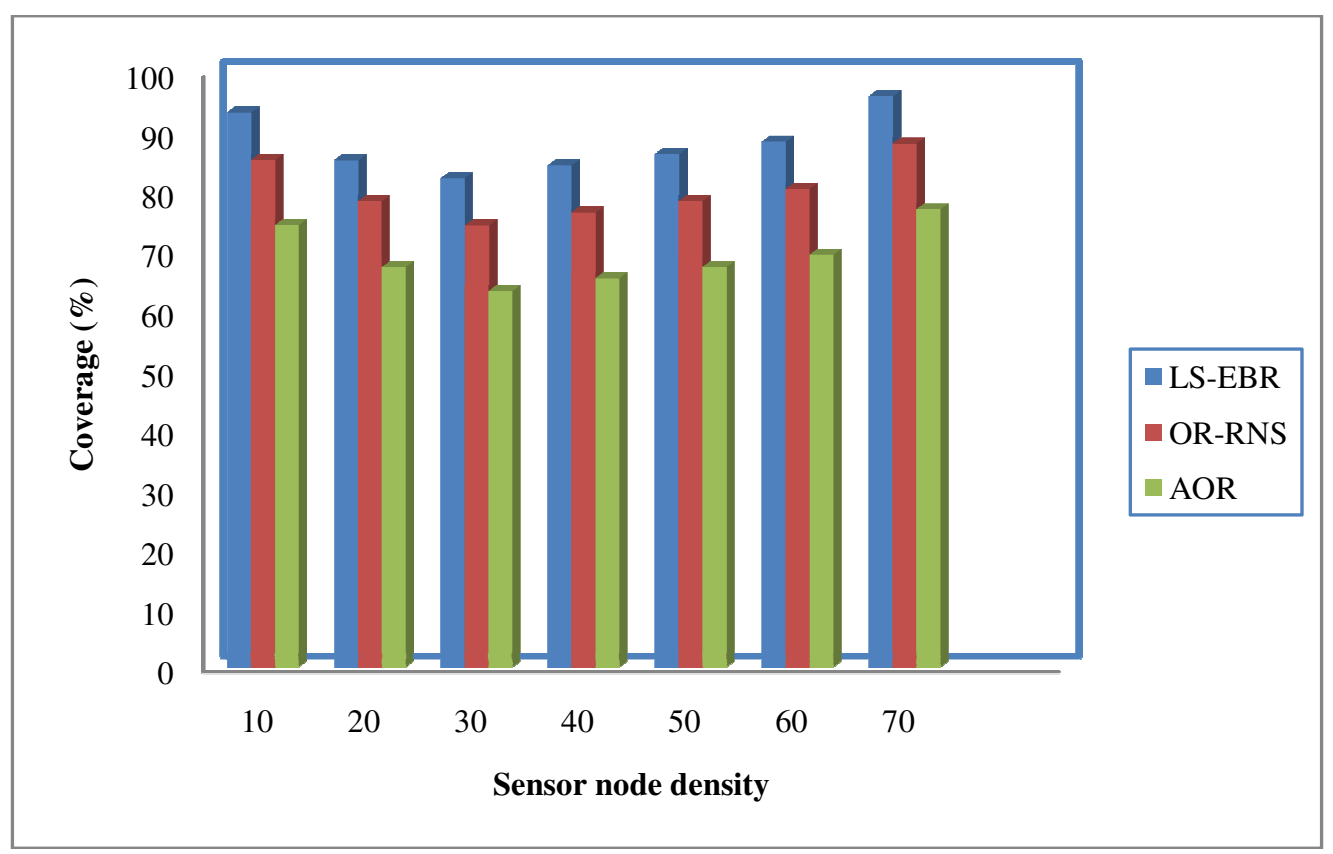

Figure 7 Coverage with different node density

The targeting results of coverage using LS-EBR model is compared with two state-of-the-art methods OR-RNS and AOR and figure 7 is presented for visual comparison based on the relevant information. The figure shows the coverage rate with respect to sensor nodes, with each sensor nodes sending different packets to the sink node. As illustrated in figure, when 10 sensor nodes sent packet to identify the route leading to the sink node, one sensor node (object) remained unmonitored using LS-EBR, two sensor node were not monitored using OR-RNS and three sensor node by applied with AOR.

Therefore, the coverage rate was observed to be improved by $8 \%$ using LS-EBR when compared to OR-RNS and $20 \%$ when compared to AOR respectively. Our routing model LS-EBR differs from the OR-RNS and AOR in that we have incorporated Enhanced Betweenness Routing algorithm. The advantage of applying Enhanced Betweenness Routing in LS-EBR is that instead of using the shortest path only, each sensor node also considers the remaining energy of sensor node into account while obtaining a route towards the sink node. This in turn improves the coverage rate of LS-EBR model by $8 \%$ compared to OR-RNS and $21 \%$ compared to AOR respectively.

For all scenarios and network patterns, average energy consumption is increasing with the data packet density. Seven unique experiments were conducted for each network size. Analysis was conducted for different set of node density $(10-70)$ with an average of 9 to 63 packets sent with a node speed $(0-8 \mathrm{~m} / \mathrm{s})$ 
International Journal on Computational Science \& Applications (IJCSA) Vol.6,No.1,February 2016

Table 3 Average energy consumption comparison

\begin{tabular}{|c|c|c|c|}
\hline \multirow{2}{*}{ Packets sent } & \multicolumn{3}{|c|}{ Average energy consumption (J) } \\
\cline { 2 - 4 } & LS-EBR & OR-RNS & AOR \\
\hline 9 & 51.35 & 54.14 & 56.21 \\
\hline 18 & 85.14 & 98.23 & 103.33 \\
\hline 27 & 110.32 & 123.14 & 128.24 \\
\hline 36 & 128.39 & 141.53 & 146.63 \\
\hline 45 & 165.14 & 179.12 & 184.22 \\
\hline 54 & 182.53 & 195.45 & 200.55 \\
\hline 63 & 198.15 & 212.34 & 217.44 \\
\hline
\end{tabular}

The results presented in figure 8 shows the average energy consumption during route identification to the sink node based on the detected objects. We can see that the values of average energy consumption increased with the decrease in the data packets sent, when the objects are sensed based on the events recorded using Poisson distribution.

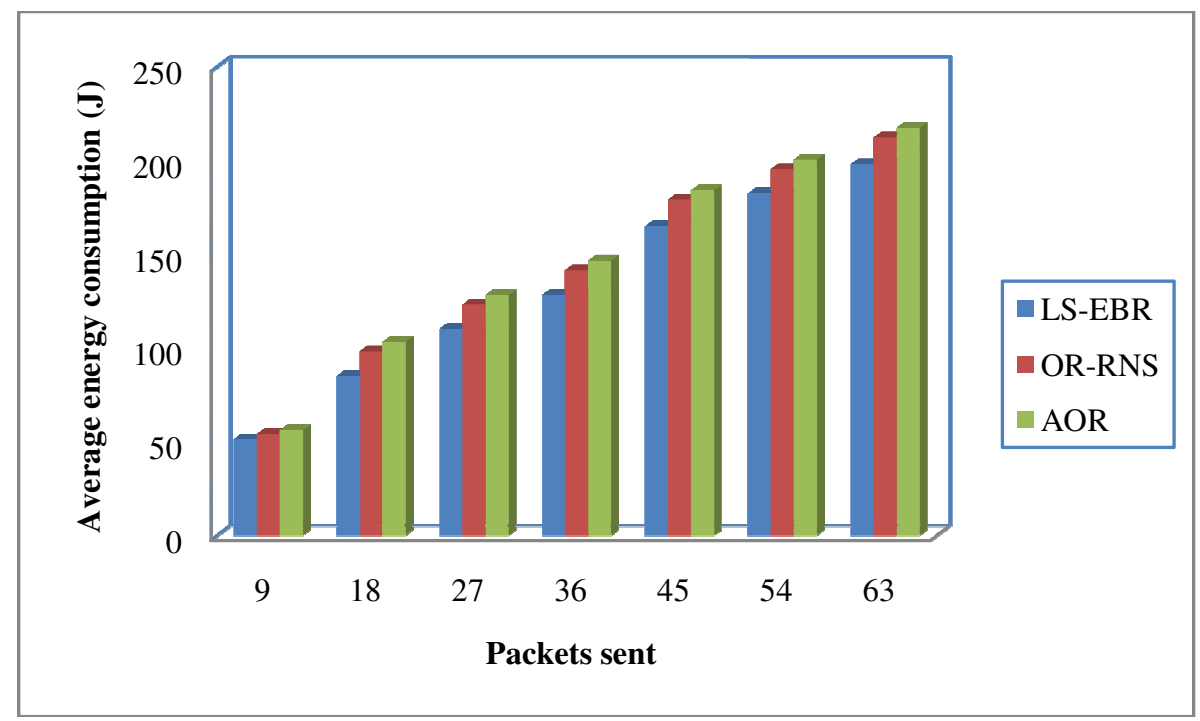

Figure 8 Average energy consumption different number of packets sent

The important observation from the figure given above is that the average energy consumption during routing is directly proportional to the number of data packets sent. Therefore though major 
International Journal on Computational Science \& Applications (IJCSA) Vol.6,No.1,February 2016

deviations are not being observed, but comparatively the LS-EBR model proved to be better. Column Difference shows the percentage difference of the particular routing scenario with respect to the average energy consumption using three different methods. The average energy consumption when 9 data packets were sent was reduced by $5 \%$ compared to OR-RNS and reduced by $9 \%$ compared to AOR. This is because of the application of Enhanced Betweenness Routing algorithm measures the average routing overhead between all pairs of vertices by restricting the length of routing overhead. This in turn helps in reducing the average energy consumption during routing using LS-EBR model by $9 \%$ compared to OR-RNS and $13 \%$ compared to AOR respectively.

\section{CONCLUSION}

Most of the sensor nodes in WSN follows different path to establish communication in the networks. Optimized routing mechanism, therefore, becomes one of major concerns in the WSN routing protocol design. In this paper, we focus on minimizing the time for obtaining the route and following efficient routing model. For this purpose, Local Search and Enhanced Betweenness Routing are designed to optimize the routing efficiency by considering the local information and routing overhead. We implement Local Search model that divert routing from frequently used sensor node. This reduces the time for routing. Next, Enhanced Betweenness Routing algorithm is designed considering both routing overhead and remaining energy of sensor node, resulting in the increase of coverage. Numerous simulation results show that the proposed solution makes significant improvement in reducing time for routing and average energy consumption by improving the coverage significantly as compared with other existing routing models.

\section{REFERENCES}

[1] A.A. Bhorkar, M. Naghshvar, T. Javidi, and B.D. Rao, "An Adaptive Opportunistic Routing Scheme for Wireless Ad-hoc Networks", IEEE/ACM Transactions on Networking, Volume 20, Issue 1, February 2012, Pages 243-256.

[2] Eric Rozner, Mi Kyung Han, Lili Qiu and Yin Zhang, "Model-driven Optimization of Opportunistic Routing”, ACM SIGMETRICS Performance Evaluation Review , Volume 39, Issue 1, June 2011, Pages 229-240.

[3] Fuzhe Zhao, You $\mathrm{Xu}$, and $\mathrm{Ru} \mathrm{Li}$, "Improved LEACH Routing Communication Protocol for a Wireless Sensor Network", Hindawi Publishing Corporation, International Journal of Distributed Sensor Networks, Volume 2012, November 2012, Pages 1-7.

[4] Henri Dubois-Ferrière, Matthias Grossglauser, and Martin Vetterli, "Valuable Detours: Least-Cost Anypath Routing”, IEEE/ACM Transactions on Networking, Volume 19, Issue 2, April 2011, Pages 333-346.

[5] Haifeng Jiang, Yanjing Sun, Renke Sun, and Hongli Xu, "Fuzzy-Logic-Based Energy Optimized Routing for Wireless Sensor Networks", Hindawi Publishing Corporation, International Journal of Distributed Sensor Networks, Volume 2013, July 2013, Pages 1-9.

[6] Jing Deng, Scott C.-H. Huang, and Yunghsiang S. Han, “An Online Relay Selection Scheme in Power Controllable Wireless Sensor Networks", Hindawi Publishing Corporation, International Journal of Distributed Sensor Networks, Volume 2012, May 2012, Pages 1-13.

[7] Juan Luo, Jinyu $\mathrm{Hu}$, Di Wu, and Renfa Li, "Opportunistic Routing Algorithm for Relay Node Selection in Wireless Sensor Networks", IEEE Transactions on Industrial Informatics, Volume 11, Issue 1, February 2015, Pages 112-12.

[8] Lei Ding, Kanke Gao, Tommaso Melodia, Stella N. Batalama, Dimitris A. Pados, and John D. Matyjas, "All-Spectrum Cognitive Networking through Joint Distributed Channelization and 
International Journal on Computational Science \& Applications (IJCSA) Vol.6,No.1,February 2016

Routing”, IEEE Transactions on Wireless Communications, Volume 12, Issue 11, November 2013, Pages 5394-5405.

[9] Luca Mottola and Gian Pietro Picco, "MUSTER: Adaptive Energy-Aware Multi-Sink Routing in Wireless Sensor Networks", IEEE Transactions on Mobile Computing, Volume 10 Issue 12, December 2011, Pages 1694-1709.

[10] Marcello Caleffi, Ian F. Akyildiz, and Luigi Paura, "OPERA: Optimal Routing Metric for Cognitive Radio Ad Hoc Networks", IEEE Transactions on Wireless Communications, Volume 11, Issue 8, August 2012, Pages 2884-2894.

[11] Moshaddique Al Ameen and Kyung-sup Kwak, "Social Issues in Wireless Sensor Networks with Healthcare Perspective", The International Arab Journal of Information Technology, Volume 8, Issue 1, January 2011, Pages 52-58.

[12] M. Mehdi Afsar, "Effective Data Aggregation Scheme for Large-scale Wireless Sensor Networks", IEEE 27th Canadian Conference on Electrical and Computer Engineering (CCECE) 2014, Pages 1-7.

[13] Rui Dai, PuWang, and Ian F. Akyildiz, "Correlation-Aware QoS Routing With Differential Coding for Wireless Video Sensor Networks", IEEE Transactions on Multimedia, Volume 14, Issue 5, October 2012, Pages 1469-1479.

[14] Shuai Wang, Anas Basalamah, Song Min Kim, Shuo Guo, Yoshito Tobe, and Tian He, "LinkCorrelation-Aware Opportunistic Routing in Wireless Networks", IEEE Transactions on Wireless Communications, Volume 14, Issue 1, January 2015, Pages 47-56.

[15] Simon S. Lam, ACM and Chen Qian, "Geographic Routing in d-dimensional Spaces with Guaranteed Delivery and Low Stretch", IEEE/ACM Transactions on Networking, Volume 21 Issue 2, April 2013, Pages 663-677.

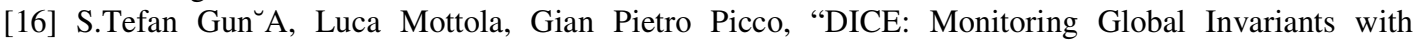
Wireless Sensor Networks", ACM Transactions on Sensor Networks (TOSN), Volume 10 Issue 4, June 2014, Pages 1-33.

[17] Vahid Ayatollahitafti, Md Asri Ngadi, Johan bin Mohamad Sharif,Mohammed Abdullahi, "An Efficient Next Hop Selection Algorithm for Multi-Hop Body Area Networks", PLoS ONE, Volume 11, Issue 1, January 2016, Pages 1-14

[18] Xiaoling Wu,Yangyang Wang, Guangcong Liu, Jianjun Li, Lei Shu, Xiaobo Zhang, Hainan Chen and Sungyoung Lee, "Energy-Efficient Routing Algorithms Based on OVSF Code and Priority in Clustered Wireless Sensor Networks", Hindawi Publishing Corporation, International Journal of Distributed Sensor Networks, Volume 2013, June 2013, Pages 1-9.

[19] Zhiguo Wan, Kui Ren, and Ming Gu, "USOR: An Unobservable Secure On-Demand Routing Protocol for Mobile Ad Hoc Networks", IEEE Transactions on Wireless Communications, Volume 11, Issue 5, May 2012, Pages 1922-1932.

\section{Authors}

M. Parthasarathi, received the B.Sc.,(Physics), M.Sc (CS) fromBharathidasan University, Trichy, in 1992 - 1998 and ME in computer science Engineering in Anna University, Coimbatore in 2008. Currently, He is pursuing the Ph.D. degree in Computer Science in MS university, Tirunelveli. His research interests wireless networks.He has more than 17 years teaching experience in various colleges. Now currently he is working in KSR college of Arts and Science, Tiruchengode

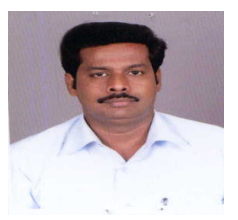
from 2005 to till date. 